\title{
Advanced Sensor Noise Analysis for CT-Scanner Identification from its 3D Images
}

\author{
Anas Kharboutly ${ }^{1}$, William Puech ${ }^{1}$, Gérard Subsol ${ }^{1}$ and Denis Hoa ${ }^{2}$ \\ ${ }^{1}$ ICAR Research Team, LIRMM, University of Montpellier/CNRS, Montpellier, France \\ kharboutly, puech, subsol@lirmm.fr \\ 2 IMAIOS, MIBI, Montpellier, France \\ denis.hoa@imaios.com
}

\begin{abstract}
Medical image processing fuses the image processing technologies in the medical disciplines. Particularly, computed tomography images provide a 3D vision of any part of the human body. These 3D images are generated by CT-Scanner devices. In this paper, we propose an advanced method of CTScanner identification from its 3D images. Basically, we analyze the sensor noise in order to identify the source CT-Scanner. For each CT-Scanner, we build three dimension identifiers regarding the three directional axes ' $X$ ', ' $Y$ ' and ' $Z$ '. The dimension identifier consists of a reference pattern noise and a correlation map. To identify the source CT-Scanner from a tested slice, we compute the correlation between each dimension identifier of each device and this tested slice. The highest correlation value represents an indicator to the source CT-Scanner and the acquisition directional axis. To isolate the pure noise, we use a wavelet based denoising algorithm. Experiments are applied on three different CT-Scanners. 10 3D images are selected from each CT-Scanner, each 3D image is composed of 512 slices. As a result, we are able to identify the acquisition CT-Scanner and the acquisition dimensional axis.
\end{abstract}

Keywords-Digital forensics, Device identification, Authentication, Pattern noise, Sensor noise, Denoising theory, Correlaion, Photo-response-non-uniformity.

\section{INTRODUCTION}

Computed tomography [1] or CT-Scan is used to generate a $3 \mathrm{D}$ representation of any part of the body. Generally, these 3D images are stored as DICOM files [2]. DICOM file consists of meta-data file and image content. Meta-data file contains all the related information about the image content. CT-Scanner identification is about finding the link between the images and its source CT-Scanner. It is considered as an important application in the absence or unauthenticated meta-data files. Especially, when these images are used in law applications, they cannot provide a strong evidence, since its header metadata file is easily modifiable. We are in need to know the source of these images in order to authenticate them. Image forensics [3], [4], [5] is the used technology to achieve this goal. One technique of image forensics is the analysis of sensor pattern noise that was first proposed by [6]. The basic component of the sensor pattern noise is the photoresponse non-uniformity (PRNU). It is the result of pixel nonuniformity, that represents the pixel sensitivity to the light [6]. PRNU is considered the most important part of the sensor pattern noise, it serves as a unique fingerprint for each device [7], [8]. The properties of PRNU that make it an ideal concept in the image forensics [9]:

- Its noise pattern is a unique one regarding each sensor.
- Each sensor has a PRNU, and all images of the same sensor inherit it.

- It is robust to most of the image processing operators.

- It is stable during the time and under different physical conditions.

Many related work about the device identification are found. Previous work in [10] and [11] studied the statistical features of images in order to identify the source camera. Similarly, a lot of other work is existed on the photo response nonuniformity (PRNU) in [6], [7], [12]. The PRNU methods construct the base of device identification. It provides a unique fingerprint for each device, this fingerprint is used to identify the devices even for the same brand and model. In addition to its simplicity, it processes the device image without need to access the device itself. In [13] the authors proposed a solution to the contamination problem, they attenuated the influence of details from the content on the fingerprint noise to improve the rate of device linking. Actually, all of these work is limited to the digital camera identification. In addition to some work on digital flatbed scanner identification, this work depends on the reference pattern noise in spatial domain [14] and the frequency domain [15].

Talking about medical device identification, very few work can be found. In [16] the authors studied the noise characteristics and compared it between two CT-Scanners of different manufactures. Another proposed method on identification is existed in [17], but it is on the primitive 2D images of radiography. Particularity, about our basic concern, the CTScanner identification, in [18] we presented a first analysis of this problem, but however, it was limited to the acquisition directional axis. We were not able to identify the images that were reformatted on ' $\mathrm{X}$ ' or ' $\mathrm{Y}$ ' directional axes.

In this paper, we present an advanced analysis of the CTScanner identification. Since the CT-Scanner images are in three dimensions, we propose an identifier for each dimensional axis. This dimension identifier is considered as a unique one, it consists of both the reference pattern noise [6], [7] and the correlation map [19], [20]. Generally, each CT-Scanner has three dimension identifiers regarding the three dimensions. The noise is basically extracted using a wavelet-based denoising algorithm, its 2D reference image is built. The correlation map gives an importance percent for each pixel of the noise reference, this importance depends on its position. Finally, the CT-Scanner and the acquisition dimensional axis are identified based on the correlation between the noise of the tested slice and the dimension identifiers. 


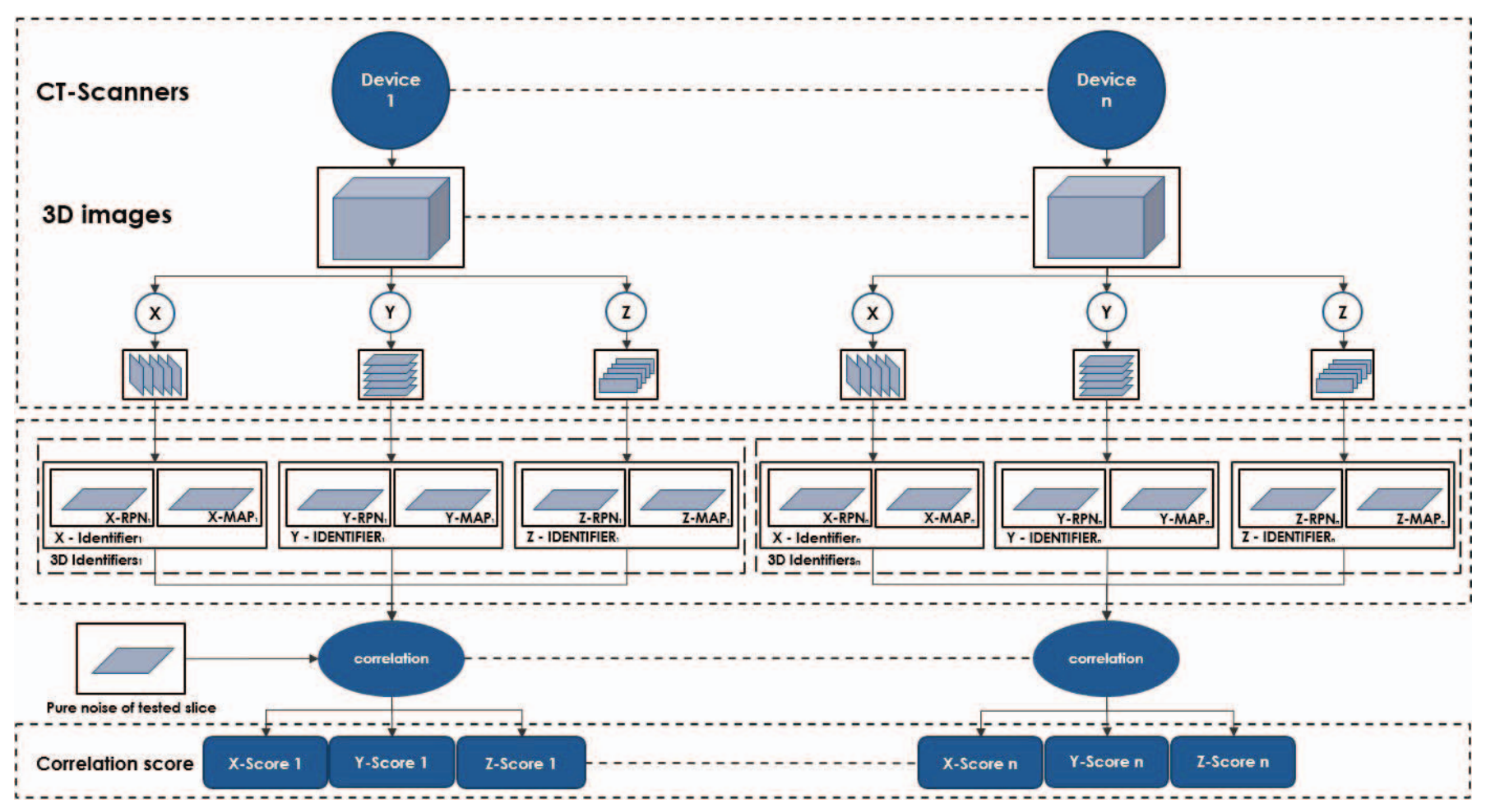

Fig. 1. Method overview.

The rest of this paper is organized as follows. In Section 2, we present our proposed method for CT-Scanner identification, dimension identifier and the identification by correlation. In Section 3, we show our experimental results in addition to some discussions. In section 4 , we conclude and provide our plan for the coming work.

\section{CT-SCANNER IdENTIFICATION Method}

The core of our proposed method is to extract the dimension identifier regarding the three directional axes of each studied CT-Scanner, as illustrated in Fig.1. In this section, we present how to build the dimension identifier of each directional axis of the CT-Scanner images. Then, how to compute both the reference pattern noise and the correlation map of each dimension identifier. Finally, how to compute the correlation between the noise component of the tested slice and the dimension identifier. How the correlation value leads us to identify both: the acquisition device and the acquisition dimensional axis.

\section{A. Dimension Identifier}

The main goal of the dimension identifier is to extract a unique fingerprint of each CT-Scanner, this fingerprint will be used to identify it later. Since the CT-Scanner devices produce 3D images, we built an identifier regarding each dimension or directional axis: ' $\mathrm{X}$ ' identifier, ' $\mathrm{Y}$ ' identifier and ' $\mathrm{Z}$ ' identifier. This dimension identifier is consisted of the reference pattern noise and the correlation map. We applied our work on a group of images regarding each device, and extracted three identifiers according to the three dimensions:
1) Reference pattern noise: Using a wavelet-based denoising algorithm described in Appendix A, we extracted the noise component of the studied image, then we suppressed the artifacts in the correction step as described in Appendix B. For each dimension identifier, we built a reference pattern noise. Generally, for each device, three references of pattern noise are built regarding the three dimensional axes. According to a specific dimension of a specific device, we selected multiple slices, these slices contain a pure noise. Then, we averaged the noise slices to generate a $2 \mathrm{D}$ reference pattern noise, that represents the approximate PRNU. This average operation removes the random noise, from the other hand it increases the PRNU noise.

$$
R P N=\frac{1}{N} \sum_{i=1}^{N} n^{(i)}
$$

where $R P N$ is the reference pattern noise, $N$ is the number of noise slices and $n$ is the noise component.

We continue the averaging to extract the three references of pattern noise of each 3D image of each tested device, RPN of 'X', RPN of 'Y' and RPN of 'Z'.

2) Correlation map: The purpose of correlation map is to give an importance percent for each pixel in the reference pattern noise, this importance comes from its position. Since the edge pixels are masked in the noise reference generation, so in the last reference noise, not all the pixel positions take the same importance, while it was served as an edge in some slices. In the correlation map, the pixel value represents its frequency as an edge in the reference noise slices: 
Table 1. Properties of the experimental images.

$$
\operatorname{map}(i, j)=\sum_{I \in R P N \text { slices }} \operatorname{mask}_{I}(i, j)
$$

where map is the edge frequencies and $\operatorname{mask}_{I}$ is the edge mask of $I$.

Now the correlation map values should be inversed to mask the edge pixels, and divided by the maximum value for normalization. Each pixel value represents its importance regarding its frequency as an edge. The pixel with high edge frequency has a lower importance, while the pixel with low edge frequency has a higher importance:

$$
\operatorname{corr} \_\operatorname{map}(i, j)=\frac{\operatorname{Inv}(\operatorname{map}(i, j))}{\max (\operatorname{map})},
$$

where corr_map is the correlation map, Inv is the inversion factor and $\max$ is the maximum value of map.

\section{B. Identification by correlation}

To test a new slice, we are in need for two information, the first one, is the acquisition CT-Scanner, and the second one, is the acquisition dimensional axis. The correlation step is applied on the three dimension identifiers of each studied device. Basically, the correlation is computed between the noise of the tested slice and the reference pattern noise. This correlation is calculated depending on the correlation map. The highest correlation value represents our guide to the source CT-Scanner and the acquisition dimensional axis:

$$
\widetilde{R P N}(i, j)=R P N(i, j) \times \operatorname{map}(i, j),
$$

here $R P N$ is the reference pattern noise and map is the correlation map.

$$
\tilde{n}(i, j)=n(i, j) \times \operatorname{map}(i, j),
$$

where $n$ is the noise component and map is the correlation map. And the correlation is:

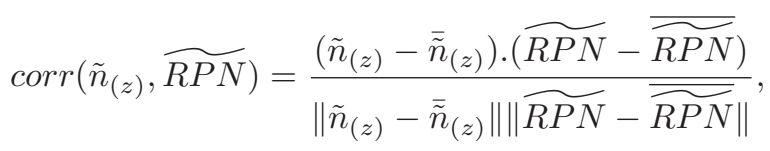

where $z$ represents the slice number.

\section{EXPERIMENTAL RESULTS}

Our experiments were applied on 10 3D images from three CT-Scanners. Each 3D image consists of 512 slices, 5120 slices of Siemens 1, 5120 slices of Siemens 2 and 5120 slices of General Electric. As a general, 15360 slices from the three CT-Scanners were used. All the images have the same parameters (Beam energy: $(120,140) \mathrm{KV}$, Pitch value: $(0.5,1)$, Reconstruction: (soft, hard)). Table III illustrates the properties of experimental images.

All of these images were reconstructed on ' $Z$ ' directional axis, we re-sampled it on the same axis to build the isotropic voxels. Then, to extract the images of ' $\mathrm{X}$ ' and ' $\mathrm{Y}$ ' dimensional axes, we reformatted each image of each device on ' $\mathrm{X}$ ' dimensional axes, then we reformatted it on ' $Y$ '. This reformatting was

\begin{tabular}{|l|l|l|l|}
\hline & Siemens 1 & Siemens 2 & GE \\
\hline Content & phantom & phantom & phantom \\
\hline Nb of images & 10 & 10 & 10 \\
\hline Nb of slices & 5120 & 5120 & 5120 \\
\hline Size (pixels) & $512 \times 512$ & $512 \times 512$ & $512 \times 512$ \\
\hline Bits per pixel & 16 & 16 & 16 \\
\hline Slice thickness & $1 \mathrm{~mm}$ & $1 \mathrm{~mm}$ & $1 \mathrm{~mm}$ \\
\hline Pixel size & $1 \mathrm{~mm}$ & $1 \mathrm{~mm}$ & $1 \mathrm{~mm}$ \\
\hline Nb of slices of RPN & 500 & 500 & 500 \\
\hline Nb of tested slices & 500 & 500 & 500 \\
\hline
\end{tabular}

basically done by rotation around the dimensional axes. Regarding each device, we selected randomly 100 slices from each image, then we repeated the same selection on each dimensional axis. Finally, we have 1000 slices of each dimensional axis. From these 1000 slices, 500 slices were used to build the dimension identifier. The rest 500 slices were used for testing.

Fig.2 illustrates an example of an original slice from a $3 \mathrm{D}$ image of phantom. This image was acquired by the first Siemens device, this figure contains the same slice from three directional axises, Fig. 3 illustrates the noise component of the same slices.
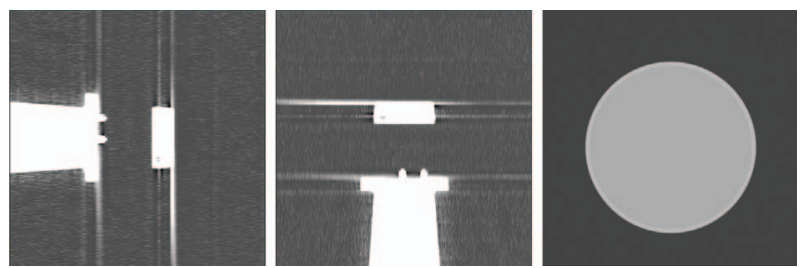

Fig. 2. Original slices from Siemens 1 ( $\mathrm{X}$ dimension, $\mathrm{Y}$ dimension, $\mathrm{Z}$ dimension).
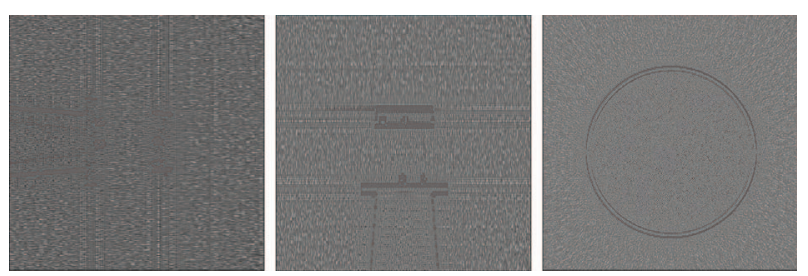

Fig. 3. Noise component from Siemens 1 ( $\mathrm{X}$ dimension, $\mathrm{Y}$ dimension, $\mathrm{Z}$ dimension).

Actually, in Fig. 3 we can notice that in addition to the noise, there are some edges or traces that rest in the slices. To remove these traces, we applied a noise correction step. In the correction step, we built an edge mask regarding each slice as illustrated in Fig. 4.

Then we applied the mask of Fig. 4 on the noise component of Fig. 3 to extract the pure noise of each slice as illustrated in Fig. 5: Then, we applied an averaging operation on the 500 selected slices, these selected slices contain noise only. Regarding each dimension, we extracted the reference pattern noise. Fig. 6 illustrates the reference pattern noise from Siemens 1 on each dimensional axis: 

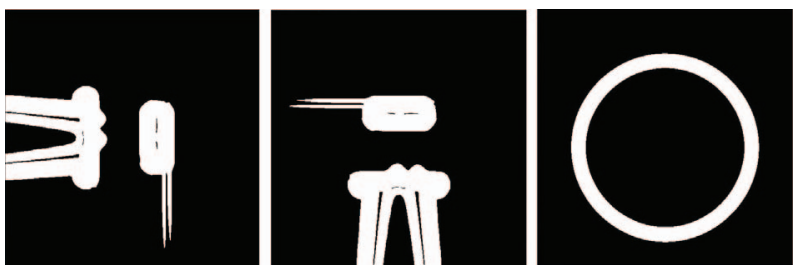

Fig. 4. Edge mask from Siemens 1 ( $\mathrm{X}$ dimension, $\mathrm{Y}$ dimension, $\mathrm{Z}$ dimension).
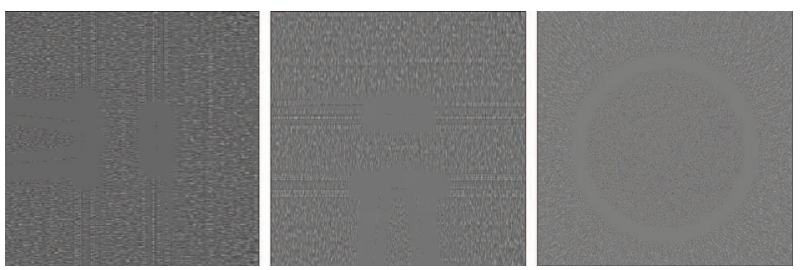

Fig. 5. Pure noise component without traces from Siemens 1 (X dimension, $\mathrm{Y}$ dimension, $\mathrm{Z}$ dimension).
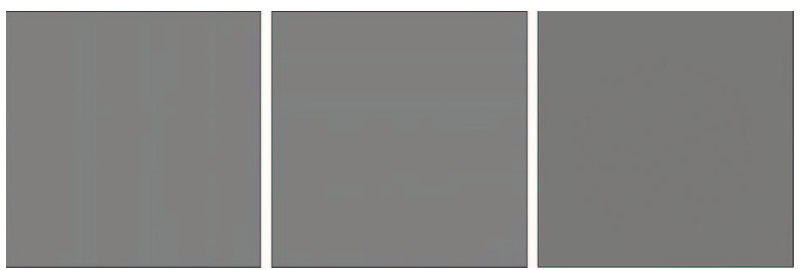

Fig. 6. Reference pattern noise from Siemens 1 (X dimension, $\mathrm{Y}$ dimension, $\mathrm{Z}$ dimension).

To build the correlation map of each directional axis of each device, we used the edge mask slices of the same ones that were used to build the reference pattern noise. Each pixel in the correlation map represents the frequency of the same pixel position in all slices of the reference pattern noise being served as an edge. Fig. 7 illustrates the correlation map of each dimensional axis from Siemens 1.
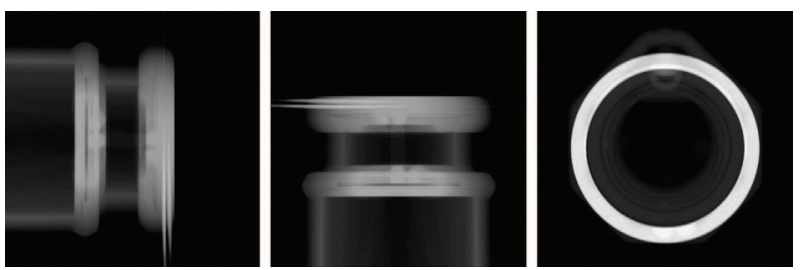

Fig. 7. The correlation map from Siemens 1 (X dimension, $\mathrm{Y}$ dimension, $\mathrm{Z}$ dimension).

We can notice that all the information in the General Electric images is centered in a circle of diameter equal to the image height or width, so we build a mask to keep a common space in all the references to apply the correlation with.

Finally, we calculated the correlation between the tested slices of each tested group and the three dimension identifiers of each device, the correlation is calculated between the noise component of the tested slice and the reference pattern noise depending on the correlation map of each dimension identifier. This correlation is computed directly, where there is almost no

\section{CPU time consumed.}

Fig.(8), (9) and (10) illustrate the correlation values between each tested group of each device and the dimension identifier of each one. In each plot, the ' $\mathrm{X}$ ' axis represents the tested slice number and the ' $\mathrm{Y}$ ' axis represents the correlation value, we can notice that the correlation values between the tested slices and the dimension identifier of the same device and the same directional axis are always the highest.

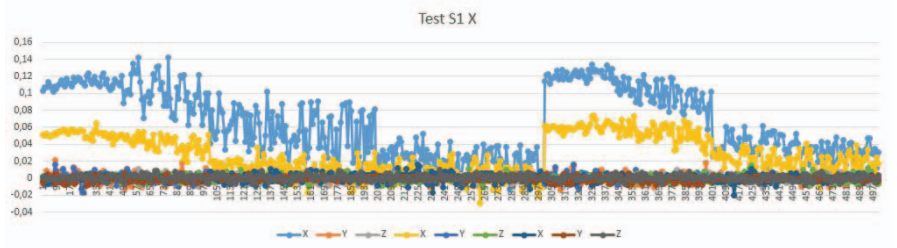

(a) Test slices from Siemens 1 and all the dimension identifiers

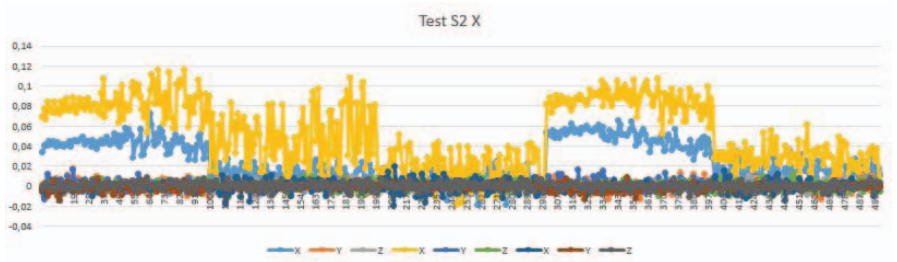

(b) Test slices from Siemens 2 and all the dimension identifiers

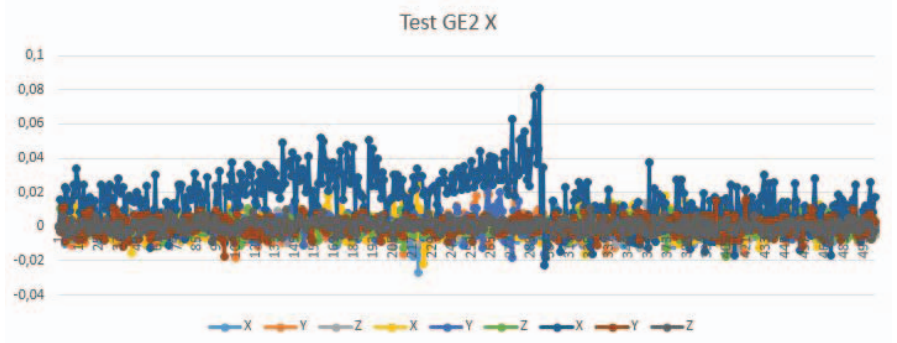

(c) Test slices from General electric and all the dimension identifiers

Fig. 8. Correlation between tested slices of ' $\mathrm{X}$ ' directional axis from the three CT-Scanners and the nine dimension identifiers of each one.

From Fig.8 and Fig.11, we notice that:

- (a) The best identification percent on ' $X$ ' axis was registered for the first device of Siemens, where $92.2 \%$ of the tested slices were correctly classified as acquired from the first Siemens, its dimensional axis was correctly identified as ' $\mathrm{X}$ '.

- (b) $88.6 \%$ of tested slices were classified correctly as acquired from Siemens 2, with correct dimensional axis.

- (c) $73 \%$ of tested slices were classified correctly as acquired from General Electric, with correct dimensional axis.

In addition, most of the tested slices on ' $\mathrm{X}$ ' axis that were not classified correctly in each tested group were associated with the same directional axis of another device:

- $5.8 \%$ of tested slices from Siemens 1 on ' $X$ ' directional axis were associated with the ' $X$ ' axis of the Siemens 2.

- $9 \%$ of tested slices from Siemens 2 on ' $X$ ' directional axis were associated with the ' $\mathrm{X}$ ' axis of the Siemens 1. 
- $6.6 \%$ of tested slices from General Electric of ' $\mathrm{X}$ ' directional axis were associated with the ' $\mathrm{X}$ ' axis of the Siemens 2.

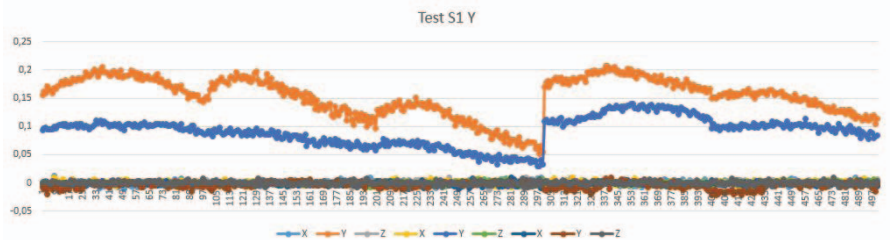

(a) Test slices from Siemens 1 and all the dimension identifiers

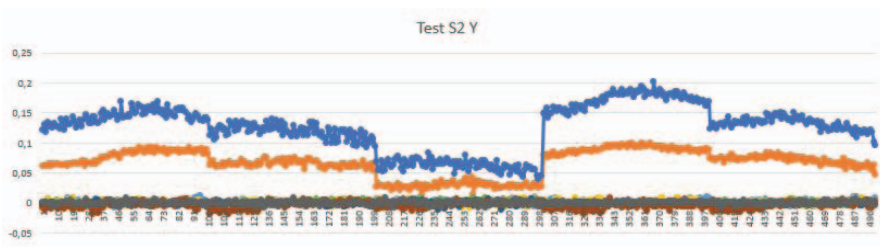

(b) Test slices from Siemens 2 and all the dimension identifiers

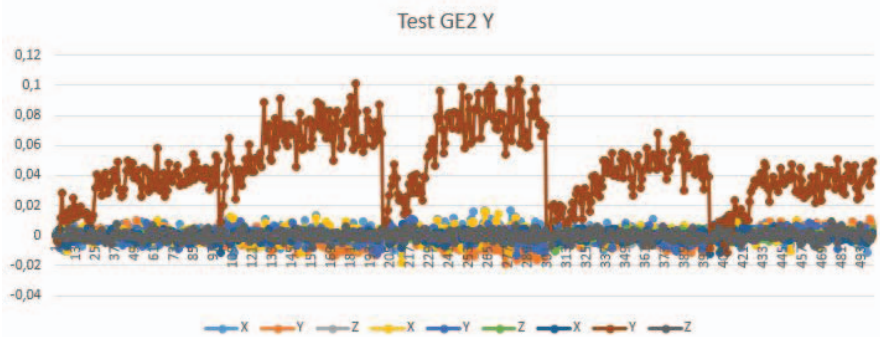

(c) Test slices from General Electric and all the dimension identifiers

Fig. 9. Correlation between tested slices of ' $\mathrm{Y}$ ' directional axis from the three CT-Scanners and the nine dimension identifiers of each one.

From Fig.9 and Fig.11, we notice that:

- The identification accuracy is $100 \%$, where the source CT-Scanner was identified correctly for all the tested slices of the first Siemens and the second one, the 'Y' directional axis was identified correctly also.

- $99.6 \%$ of tested slices were classified correctly as acquired from General Electric, and its directional axis was 'Y'.

From Fig.10 and Fig.11, we notice that the identification accuracy is $100 \%$, where the source CT-Scanner was identified correctly for the three CT-Scanners on ' $Z$ ' directional axis.

From Fig.11 also, we noticed that the identification on ' $Y$ ' and ' $\mathrm{Z}$ ' axis, generally, is more accurate than ' $\mathrm{X}$ ' axis.

\section{CONCLUSION AND FUTURE WORK}

In this paper, we proposed an advanced analysis of the sensor pattern noise, that is used to identify the CT-Scanner. Our experiments show the strong ability of our proposed method to identify the source CT-Scanner, the acquisition directional axis and the source CT-Scanner for any slice from whatever directional axis.

In the coming work, we will study the influence of acquisition parameters and image compression on our proposed

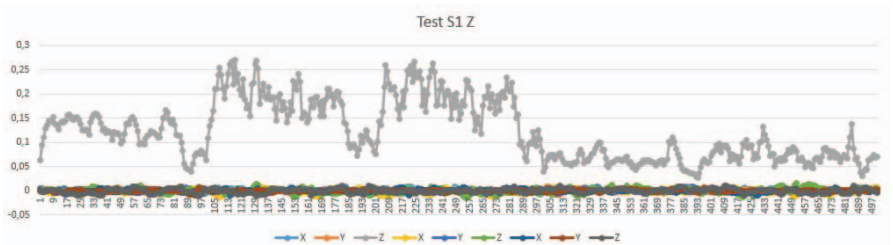

(a) Test slices from Siemens 1 and all the dimension identifiers Test $52 Z$

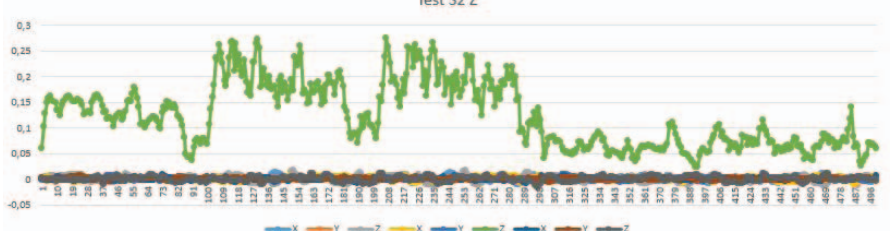

(b) Test slices from Siemens 2 and all the dimension identifiers

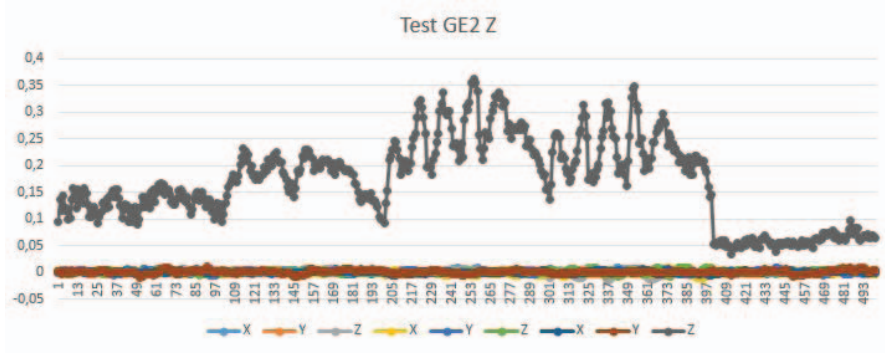

(c) Test slices from General Electric and all the dimension identifiers

Fig. 10. Correlation between tested slices of ' $Z$ ' directional axis from the three CT-Scanners and the nine dimension identifiers of each one.

\begin{tabular}{|c|c|c|c|c|c|c|c|c|c|}
\hline Ref \ Test & S1X & S1Y & $\$ 12$ & $52 x$ & S2Y & $\$ 22$ & GEX & GEY & GEZ \\
\hline $51 x$ & $92.2 \%$ & 0 & 0 & $9 \%$ & 0 & 0 & $3.4 \%$ & 0 & 0 \\
\hline SIY & $0.2 \%$ & $100 \%$ & 0 & $0.2 \%$ & 0 & 0 & $1.8 \%$ & 0 & 0 \\
\hline 512 & 0 & 0 & $100 \%$ & $0.2 \%$ & 0 & 0 & $3.0 \%$ & 0 & 0 \\
\hline $52 x$ & $5.8 \%$ & 0 & 0 & $88.6 \%$ & 0 & 0 & $6.6 \%$ & 0 & 0 \\
\hline $52 \mathrm{Y}$ & 0 & 0 & 0 & 0 & $100 \%$ & 0 & $0.8 \%$ & $0.2 \%$ & 0 \\
\hline$\$ 2 Z$ & $0.6 \%$ & 0 & 0 & $0.6 \%$ & 0 & $100 \%$ & $4.2 \%$ & 0 & 0 \\
\hline GEX & $0.4 \%$ & 0 & 0 & $0.8 \%$ & 0 & 0 & $73 \%$ & $0.2 \%$ & 0 \\
\hline GEY & $0.4 \%$ & 0 & 0 & 0 & 0 & 0 & $4.4 \%$ & $99.6 \%$ & 0 \\
\hline GEZ & $0.2 \%$ & 0 & 0 & 0.4 & 0 & 0 & $2.2 \%$ & 0 & $100 \%$ \\
\hline
\end{tabular}

Fig. 11. Identification accuracy

method.

\section{APPENDIX}

\section{A. Denoising algorithm}

In the frequency domain, we apply a Wiener filter based wavelet transformation [21], [22], [23]. Basically, this algorithm is composed of two parts. First, local variance estimation of the wavelet components. Second, denoising these components using Wiener filter [24] as follows:

- Compute four levels of wavelet decomposition of the original image. In each level, mark out the three high frequency sub-bands that are horizontal, vertical and diagonal. For four levels of wavelet decomposition with three sub-bands in each level we have 12 sub-bands for each processed slice.

- For each wavelet sub-band, based on the pixel neighborhood with four levels. From the first boundary neighbors 
with square size of $(3 \times 3)$ to the fourth boundary ones with square size of $(9 \times 9)$, we apply the local variance estimation:

$$
\hat{\sigma}_{W}^{2}(i, j)=\max \left(0, \frac{1}{W^{2}} \sum_{(i, j) \in W * W}\left(X^{2}(i, j)-\sigma_{0}^{2}\right)\right),
$$

where $W \in\{3,5,7,9\}$ refers to the neighborhood size, $X$ is the wavelet sub-band and $\sigma_{0}$ is an initial integer constant value that we tuned manually, $\sigma_{0} \in[1,6]$. Among the four previous values regarding the four levels of neighborhood, we select the minimum value as the estimated variance:

$$
\hat{\sigma}^{2}(i, j)=\min \left(\sigma_{3}^{2}(i, j), \sigma_{5}^{2}(i, j), \sigma_{7}^{2}(i, j), \sigma_{9}^{2}(i, j)\right),
$$

- Denoise the wavelet sub-bands using Wiener filter, that is a minimum mean square error filter. It has the capabilities of handling both the degradation function as well as the noise that has corrupted the signal:

$$
X_{d e n}(i, j)=X(i, j) \frac{\hat{\sigma}^{2}(i, j)}{\hat{\sigma}^{2}(i, j)+\sigma_{0}^{2}},
$$

where $X$ is the wavelet sub-band.

- Apply the inverse wavelet transformation on the denoised wavelet sub-bands to get the denoised component $F(s)$ of the original image $s$.

\section{B. Noise extraction and correction}

To extract the noise, we apply a subtraction between the original slice and the denoised one that is a result of the denoising method.

$$
n^{(i)}=s^{(i)}-F\left(s^{(i)}\right),
$$

where $n$ is the noise component, $s$ is the slice, $F()$ is the denoising function and $i$ is the slice number.

As a result of the subtraction operation, we get the noise component, but in addition to the noise, there exist another traces or edges that rest in the noise image. To get deal with this kind of traces, we apply the noise correction step, we apply an edges detection on the original image in order to extract the edges that exist in the slice [14] as follow:

- Apply a blurring filter to remove the noise.

- Compute the gradient of each pixel.

- Compute the norm of the gradient for each pixel and create the image of the values.

- Finally, threshold the norm image to extract the maximum local value that represents the edges mask.

Finally, we apply the mask of edges detected image on the image of subtraction result to get the pure noise image without any traces or edges.

\section{REFERENCES}

[1] J. T. Bushberg and J. M. Boone, The essential physics of medical imaging, Lippincott Williams \& Wilkins, 2011.
[2] K. D. Toennies, Guide to Medical Image Analysis - Methods and Algorithms, Advances in Computer Vision and Pattern Recognition. Springer, 2012.

[3] J. Redi, W. Taktak, and J. L. Dugelay, "Digital image forensics: a booklet for beginners," Multimedia Tools and Applications, vol. 51, no. 1, pp. 133-162, 2011.

[4] A. Piva, "An overview on image forensics," ISRN Signal Processing, vol. 2013, 2013.

[5] H. T. Sencar and N. Memon, Digital Image Forensics: There is More to a Picture Than Meets the Eye, Springer, 2013.

[6] J. Lukas, J. Fridrich, and M. Goljan, "Digital camera identification from sensor pattern noise," IEEE Transactions on Information Forensics and Security, vol. 1, no. 2, pp. 205-214, 2006.

[7] M. Chen, J. Fridrich, M. Goljan, and J. Lukás, "Determining image origin and integrity using sensor noise," Information Forensics and Security, IEEE Transactions on, vol. 3, no. 1, pp. 74-90, 2008.

[8] M. Chen, J. Fridrich, and M. Goljan, "Digital imaging sensor identification (further study," in In Security, Steganography, and Watermarking of Multimedia Contents IX. Edited by Delp, Edward J., III; Wong, Ping Wah. Proceedings of the SPIE, Volume 6505, 2007.

[9] H. B. COSTA, R. F. ZAMPOLO, D. M. CARMO, A. R. CASTRO, and E. P. SANTOS, "On the practical aspects of applying the prnu approach to device identification tasks," International Conference on Multimedia Forensics, Surveillance and Security, September 2012.

[10] O. Celiktutan, İ. Avcibaş, B. Sankur, and N. Memon, "Source cellphone identification," IEEE Signal Processing and Communications Applications, pp. 1-3, April 2006.

[11] M. Kharrazi, H.T. Sencar, and N. Memon, "Blind source camera identification," in Image Processing, 2004. ICIP '04. 2004 International Conference on, Oct 2004, vol. 1, pp. 709-712 Vol. 1.

[12] X. Kang, Y. Li, Z. Qu, and J. Huang, "Enhancing source camera identification performance with a camera reference phase sensor pattern noise," Information Forensics and Security, IEEE Transactions on, vol. 7, no. 2, pp. 393-402, April 2012.

[13] C. T. Li, "Source camera identification using enhanced sensor pattern noise," Trans. Info. For. Sec., vol. 5, no. 2, pp. 280-287, 2010.

[14] N. Khanna, A. K. Mikkilineni, G. T.-C. Chiu, J. P. Allebach, and E. J. Delp, "Scanner identification using sensor pattern noise," in SPIE Conference on Security, Steganography, and Watermarking of Multimedia Contents, Edward J. Delp and Ping Wah Wong, Eds., 2007, vol. 6505 .

[15] C.-H. Choi, M.-J. Lee, and H.-K. Lee, "Scanner identification using spectral noise in the frequency domain," in Image Processing (ICIP), 2010 17th IEEE International Conference on, Sept 2010, pp. 2121-2124.

[16] J. B. Solomon, O. Christianson, and E. Samei, "Quantitative comparison of noise texture across CT scanners from different manufacturers," Medical physics, vol. 39, no. 10, pp. 6048-55, October 2012.

[17] Y. Duan, G. Coatrieux, and H. Shu, "Identification of digital radiography image source based on digital radiography pattern noise recognition," in Image Processing (ICIP), 2014 IEEE International Conference on IEEE, 2014, pp. 5372-5376.

[18] A. Kharboutly, W. Puech, G. Subsol, and D. Hoa, "Ct-scanner identification based on sensor noise analysis," in Visual Information Processing (EUVIP), 2014 5th European Workshop on. IEEE, 2014, pp. 1-5.

[19] C. Shi, N.-F. Law, H.-F. Leung, and W.-C. Siu, "Weighting optimization with neural network for photo-response-non-uniformity-based source camera identification," in Asia-Pacific Signal and Information Processing Association, 2014 Annual Summit and Conference (APSIPA), Dec 2014, pp. 1-7.

[20] L.-H. Chan, N.-F. Law, and W.-C. Siu, "A two dimensional camera identification method based on image sensor noise," in Acoustics, Speech and Signal Processing (ICASSP), 2012 IEEE International Conference on, March 2012, pp. 1741-1744.

[21] M.K. Mihçak, I Kozintsev, and K. Ramchandran, "Spatially adaptive statistical modeling of wavelet image coefficients and its application to denoising," in Acoustics, Speech, and Signal Processing, 1999. Proceedings., 1999 IEEE International Conference on, Mar 1999, vol. 6, pp. 3253-3256.

[22] E. Jerhotová, A. Procházka, and J. Švihlík, Biomedical image volumes denoising via the wavelet transform, INTECH Open Access Publisher, 2011.

[23] P. Gravel, G. Beaudoin, and J. A. De Guise, "A method for modeling noise in medical images," Medical Imaging, IEEE Transactions on, vol. 23, no. 10, pp. 1221-1232, 2004.

[24] N. Jacob and A. Martin, "Image denoising in the wavelet domain using Wiener filtering," Unpublished course project, 2004, [Online], Project Report, Available: http://homepages.cae.wisc.edu/ ece533/project/ f04/jacob_martin.pdf. 